PAPER • OPEN ACCESS

Combined thermomechanical and optical simulations of planar-optical polymer waveguides

To cite this article: Monali Suar et al 2020 J. Opt. 22125801

View the article online for updates and enhancements.

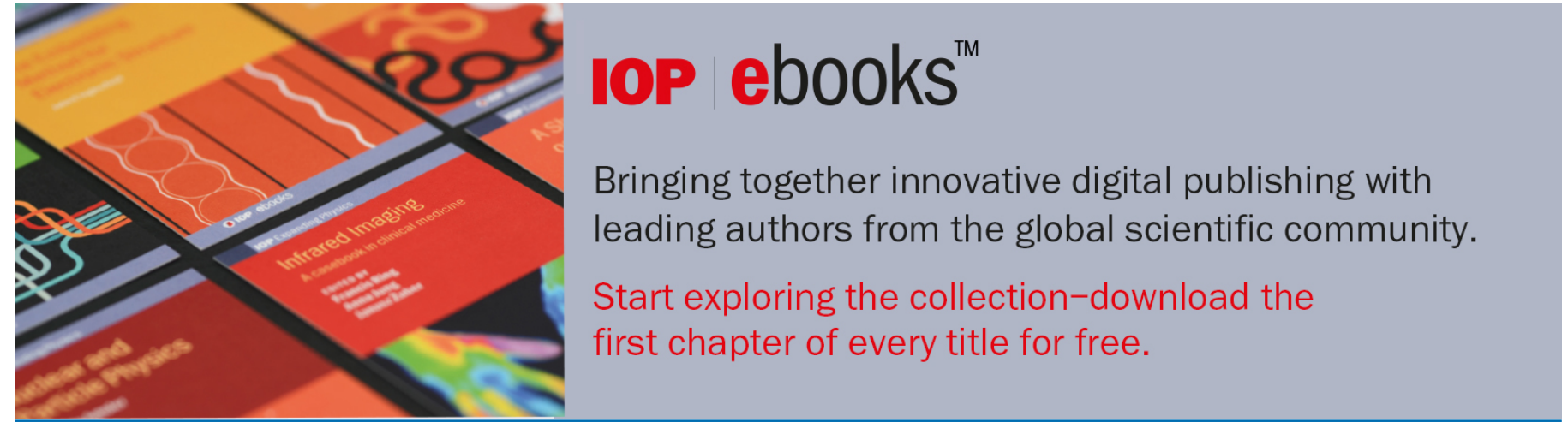

This content was downloaded from IP address 194.95 .157 .32 on 12/03/2021 at 10:14 


\title{
Combined thermomechanical and optical simulations of planar-optical polymer waveguides
}

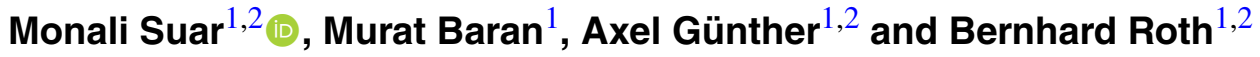 \\ ${ }^{1}$ Hannover Centre for Optical Technologies, Leibniz Universität Hannover, Nienburger Str. 17, Hannover \\ 30167, Germany \\ ${ }^{2}$ Cluster of Excellence PhoenixD (Photonics, Optics, and Engineering - Innovation Across Disciplines), \\ Hannover 30167, Germany \\ E-mail: monali.suar@hot.uni-hannover.de
}

Received 18 June 2020, revised 22 August 2020

Accepted for publication 13 October 2020

Published 29 October 2020

\begin{abstract}
In this work, we describe a theoretical approach for combined thermal, mechanical and optical simulation and analysis of planar polymer waveguides. We consider a finite element approach for thermal and stress/deformation simulation. Also, a Crank-Nicholson finite difference beam propagation method (CN-BPM) is implemented to perform the optical simulation. The results of the finite element (thermo-mechanical) analysis are coupled with the CN-BPM results to carry out the optical simulation of poly(methyl methacrylate) (PMMA) waveguides as function of temperature. For thermal simulation, a model was designed where a polysilicon microheater was added to the upper cladding of the PMMA waveguides to vary the temperature between $20{ }^{\circ} \mathrm{C}$ and $200{ }^{\circ} \mathrm{C}$. Thus, the impact of the induced temperature gradients on the refractive index modulation of the PMMA waveguides and the corresponding change in numerical aperture are obtained. In addition, the temperature gradients influence the beam intensity profiles and the movement of the primary eyes within the optical waveguides, thus, impacting the optical properties. Furthermore, the thermally induced mechanical stress and deformation were calculated for transverse and axial directions. In the next step, validation of the model by systematic experimental studies will be performed. In general, our approach provides a toolbox for more comprehensive multi-physics theoretical analysis of polymer-optical waveguides which, in future, can be extended to more complex and functional structures as required for flexible sensor networks, as example.
\end{abstract}

Keywords: polymer waveguides, optical components and networks, multi-physics simulation and analysis

(Some figures may appear in colour only in the online journal)

\section{Introduction}

Original content from this work may be used under the terms of the Creative Commons Attribution 4.0 licence. Any further distribution of this work must maintain attribution to the author(s) and the title of the work, journal citation and DOI.
Polymer waveguide structures lend themselves as promising components for broadband telecommunication, i.e. for optical network and computing systems, or sensor networks. All these systems rely on the advancement of optical waveguide manufacturing technology to produce low-loss waveguides and interconnect as well as methods for their 
comprehensive analysis taking into account all relevant physical effects. So far, many photonic devices such as thermal optical switches (TOSs) [1,2], variable optical attenuators (VOAs) [3, 4], optical couplers/splitters [5-7], and arrayed waveguide gratings (AWGs) [8,9] were fabricated by employing polymer waveguides. The design and fabrication of a polymer transmission path for communication and sensing application is demonstrated in [10]. Also, the polymer based photonic components integrated to photonic circuits for next generation datacenter systems were reviewed [11]. A simple and reliable technique to fabricate optical interconnects also exploits the self-writing of the waveguides in photosensitive and photopolymer media [12-14]. Another simple and cost efficient fabrication technique for planar polymer optical waveguide through hot embossing and doctor blading techniques was investigated by Rezem et al $[15,16]$. A detailed fabrication of waveguide biosensors by the dip-floating technique was demonstrated in [17]. The poly(methyl methacrylate) (PMMA) grating structures are realized using the femtosecond laser writing technique in [18]. The investigation on photochemical mechanism and the nonlinear photo-absorptive effect on dry acrylamide/polyvinyl alcohol based photopolymer was analyzed by Li et al [19]. Polymers are, in general, attractive for integrated optical devices as they enable rapid processability, cost-effectiveness, high yield, and efficient optical performance, i.e. relatively low optical losses and smaller birefringence compared to their silica counterparts. Furthermore, polymers allow for power-efficient thermal actuation due their large negative thermo-optic coefficients $\left(\frac{d n}{d T}=-\left(1\right.\right.$ to $\left.4 * 10^{-4}{ }^{\circ} \mathrm{C}^{-1}\right)$ which can be $10-40$ times higher than in more conventional optical materials such as glass [20]. The thermo-optic coefficients play a vital role in determining and assessing the performance of photonic polymer devices, as detailed by Zhang et al [21]. On the other hand, the operating conditions may easily drift and lead to a change in refractive index, induced by the change in the environmental conditions during the field service, causing thermal and functional instabilities in such devices. The change in refractive index with respect to the temperature mostly depends on two counteracting effects, i.e. the increase in polarizability with temperature and the change in density of the polymer caused by the positive thermal expansion coefficient. A small thermally induced change of refractive index can significantly alter the light intensity distribution within waveguides, especially in coupled systems [22]. By using microheaters, temperature gradients can practically be induced within the waveguides, which in turn is used to switch the light paths [22]. The service temperature of polymers is limited to the range between $60{ }^{\circ} \mathrm{C}$ and $250{ }^{\circ} \mathrm{C}$, which is substantially less than for optical glasses $\left(400{ }^{\circ} \mathrm{C}-700{ }^{\circ} \mathrm{C}\right)$ [23]. Also, the heat and stress generated in the polymer waveguides will eventually lead to mechanical deformations as well. Compressive and tensile stress are introduced in the polymer waveguide either parallel or perpendicular to the beam propagation directions. This is mainly caused due to the difference in thermal expansion coefficients between the core and cladding of the material. The change in the environmental conditions thus represents a major drawback for the usability of polymer materials in this field.

In this article, we focus on combining thermal, mechanical and optical simulations of polymer waveguide structures as basic elements for polymer based photonic devices to more comprehensively analyse their properties and performance and take into account the relevant physical effects. We theoretically investigate the variation of refractive index of optically transparent PMMA waveguides within the temperature range from $20{ }^{\circ} \mathrm{C}$ to $200{ }^{\circ} \mathrm{C}$ which is a reasonable [24, 25]. Alongside, optical simulations of beam intensity profiles within the PMMA waveguides at different temperatures induced by the microheaters are presented. The intensity losses during the transmission and the normalized output power from the exit port are calculated to quantify the combined influence on waveguide geometry, refractive index and numerical aperture. Furthermore, the mechanical deformation generated by induced thermal loads in transverse and axial beam direction are determined. In general, we investigated the relatively simple case where a change in refractive index of the polymer waveguide was induced by the change in the temperature of the microheater. This, in turn, lead to the variation in beam intensities carried by waveguide. Also, the mechanical stress built up due to the heat producing elongations of the waveguide dimensions was studied. This approach to multi-physics simulations can thus be applied to test the influence of environmental instabilities on similar systems, i.e. thermo-optic switches or complex polymer-optical structures for sensor networks.

\subsection{Finite element thermo-mechanical simulation}

The finite element thermo-mechanical simulation and analysis of PMMA polymer waveguides are carried out by using the FEATool that combines a multi-physics and partial differential equation (PDE) simulation environment and allows for modeling of heat transfer, fluid dynamics, chemical engineering, structural mechanics and electromagnetic effects [26]. The modeling process is divided into sequential sub-processes such as definition of the geometry, the generation of the mesh/grid, and the specification of the physical effects and boundary conditions, among others [27]. These processes are often iterative in nature. Also, one may repeat some of the procedure steps to achieve an optimal performance for the designed model. In our approach, we simultaneously used the heat transfer module and plain stress analysis module of the tool under the multiphysics mode to carry out the finite element simulations of the waveguides. The model structures were designed in two dimensions so that they can be easily coupled to our 2-dimensional finite difference based beam propagation method (BPM) used for the optical simulation. Thus, the simulations do not require large memory space. We selected a fast and efficient triangular grid for mesh generation as it allows more flexibility in specifying the grid size for different subdomains of the geometry and boundary regions. In our case, the heat transfer by the conduction mechanism is considered. With respect to thermal analysis, the effect of 
Table 1. Model parameter values for thermal simulation.

\begin{tabular}{lrcc}
\hline & $\rho\left(\mathrm{kg} \mathrm{m}^{-3}\right)$ & $C_{p}\left(\mathrm{~J} / \mathrm{kg}^{* \circ} \mathrm{C}\right)$ & $k\left(\mathrm{~W} / \mathrm{m}^{* \circ} \mathrm{C}\right)$ \\
\hline Monomer & 940 & 1890 & 0.147 \\
PMMA & 1190 & 1500 & 0.25 \\
Microheater (polysilicon) & 2320 & 678 & 34 \\
\hline
\end{tabular}

radiation and convection in the polymer waveguide can be neglected [28]. For the thermal simulations, we used the following equation [29]:

$$
\rho C_{\mathrm{p}} T^{\prime}+\nabla \cdot(-k \nabla T)=Q .
$$

Here, $\rho$ is the density, $C_{\mathrm{p}}$ the specific heat and $k$ the thermal conductivity of the PMMA material. $T$ is the temperature distribution within the waveguide and $T^{\prime}$ its derivative with respect to time. The detailed values of the parameters used for the simulations are provided in table 1. Furthermore, to calculate the deformation (elongation) in the waveguides the plane stress equation was used:

$$
\rho u^{\prime}-E /\left(1-\nu^{2}\right)\left(\left(u_{x}+\nu v_{y}(1+\nu) \alpha T\right)+(1-\nu) / 2\left(u_{y}+v_{x}\right)\right)=0,
$$

$$
\rho \nu^{\prime}-E /\left(1-\nu^{2}\right)\left(\left(\nu u_{x}+v_{y}(1+\nu) \alpha T\right)+(1-\nu) / 2\left(u_{y}+v_{x}\right)\right)=0 \text {. }
$$

Here, $E$ is the Young's modulus, $v$ is the Poisson's ratio and $\alpha$ is the thermal expansion coefficient. and $v$ are the displacements along the $x$-axis and $y$-axis, i.e. material density, $u$ perpendicular to the optical axis of the waveguide, respectively.

\subsection{Beam propagation based optical simulation}

To calculate the light distribution within PMMA waveguide core, a scalar two-dimensional beam propagation method was implemented, inspired by the work of Pedrola [30]. The slowly varying field envelope is assumed and then the paraxial approximation to the Helmholtz equation is applied to obtain the beam propagation equation as shown below:

$$
2 i n_{0} k_{o} \frac{\mathrm{d} u(x, z)}{\mathrm{d} z}=\frac{\mathrm{d}^{2} u(x, z)}{\mathrm{d}^{2} x}+k_{0}^{2}\left(n^{2}-n_{0}^{2}\right) u(x, z) .
$$

A Crank-Nicholson based finite difference scheme is applied to equation (4) to obtain the numerical solution, which is non-local in time. Here, $n_{0}$ is the initial average value for the refractive index of the homogenous media. The current value of refractive index is $n$, which is a dynamic value and is calculated from the thermal distribution within the PMMA sample, as shown below. The free space wavenumber is $k_{0}$ and calculated from the wavelength $(\lambda)$ of the input Gaussian beam via $k_{0}=2 * \pi / \lambda$. The electromagnetic field amplitude is denoted by $u(x, z) . x$ and $z$ are the transverse and axial beam directions, respectively. The waist of the Gaussian beam is assumed to illuminate the PMMA sample from the left input facet and the output beam is collected from the right exit facet. Along with the CN-BPM transparent boundary conditions (TBC) are employed to equation (4) to carry out the optical simulations [31]. Because of lower computational complexity and reduced computational time, the CN-BPM was considered to carry out the optical simulations of the waveguides. In general, our approach can also be combined with the more accurate finite difference time domain (FDTD) method for the optical part.

\section{Results and discussions}

Figure 1 shows the schematics of the cross-sectional view of the planar PMMA waveguide that was further considered for the thermo-mechanical analysis along with the optical simulations. The core part of the waveguide is assumed to be PMMA polymer material, whereas the upper/lower cladding of the waveguide is considered to be from monomer in liquid form. In general, polymers can be used for thermo-optical switches, due their large negative thermo-optic coefficients. To mimic this case, we added a polysilicon microheater to the model. As the position and the dimension of the microheater have a significant impact on the efficiency of the thermo-optic (TO) waveguide switch and the switching time, the microheater was placed at two different positions, on the top and inside the upper waveguide cladding, respectively. In the first case, the polysilicon microheater was placed on the top of the upper cladding; see figure 1(a). Here, the width of the core and cladding part of the waveguide along the transverse $x$ - (and $y$-) axis were $40 \mu \mathrm{m}$ and $20 \mu \mathrm{m}$, respectively. The length along the propagation $z$-axis of the PMMA waveguide was $1600 \mu \mathrm{m}$ (figure 1(a)). The polysilicon microheater in figure 1(a) had a width of $20 \mu \mathrm{m}$ along the $x$-axis and a length of $1200 \mu \mathrm{m}$ along the $z$-axis. In the second case, the microheater with the same dimensions was buried in the middle part of the upper cladding. In figure 1(b), the waveguide core was again considered to be PMMA material with a width of $40 \mu \mathrm{m}$ along the transverse $x$ - (and $y$-) axis and a length of $1600 \mu \mathrm{m}$ along the $z$-axis. The upper/lower claddings were again monomer material with a width of $80 \mu \mathrm{m}$ along the $x$ - (and $y$-) axis. The microheater was placed at a distance of $30 \mu \mathrm{m}$ from the core part in figure 1(b). The width of the waveguide core is the same for both models shown in figures 1(a) and (b). The cladding width in figure 1(b) is different compared to figure 1(a) in order to fit the top microheater within the upper cladding of the waveguide. The optical axis of the waveguides, the central line L1, passes through the middle section of the core along the $z$-direction and was considered further to obtain the induced elongation. Using these pre-defined geometries, the thermal simulations were performed by using the already described heat transfer module. The initial temperature distribution was $T_{0}=20{ }^{\circ} \mathrm{C}$ for the whole waveguide. Then, the temperature of the polysilicon heater was varied from the initial temperature $T_{0}$ to the maximum temperature $T_{\max }=200^{\circ} \mathrm{C}$. Thereby, the output intensity from the exit port of the waveguide dropped below $50 \%$ of the initial input beam intensity. Appropriate boundary 


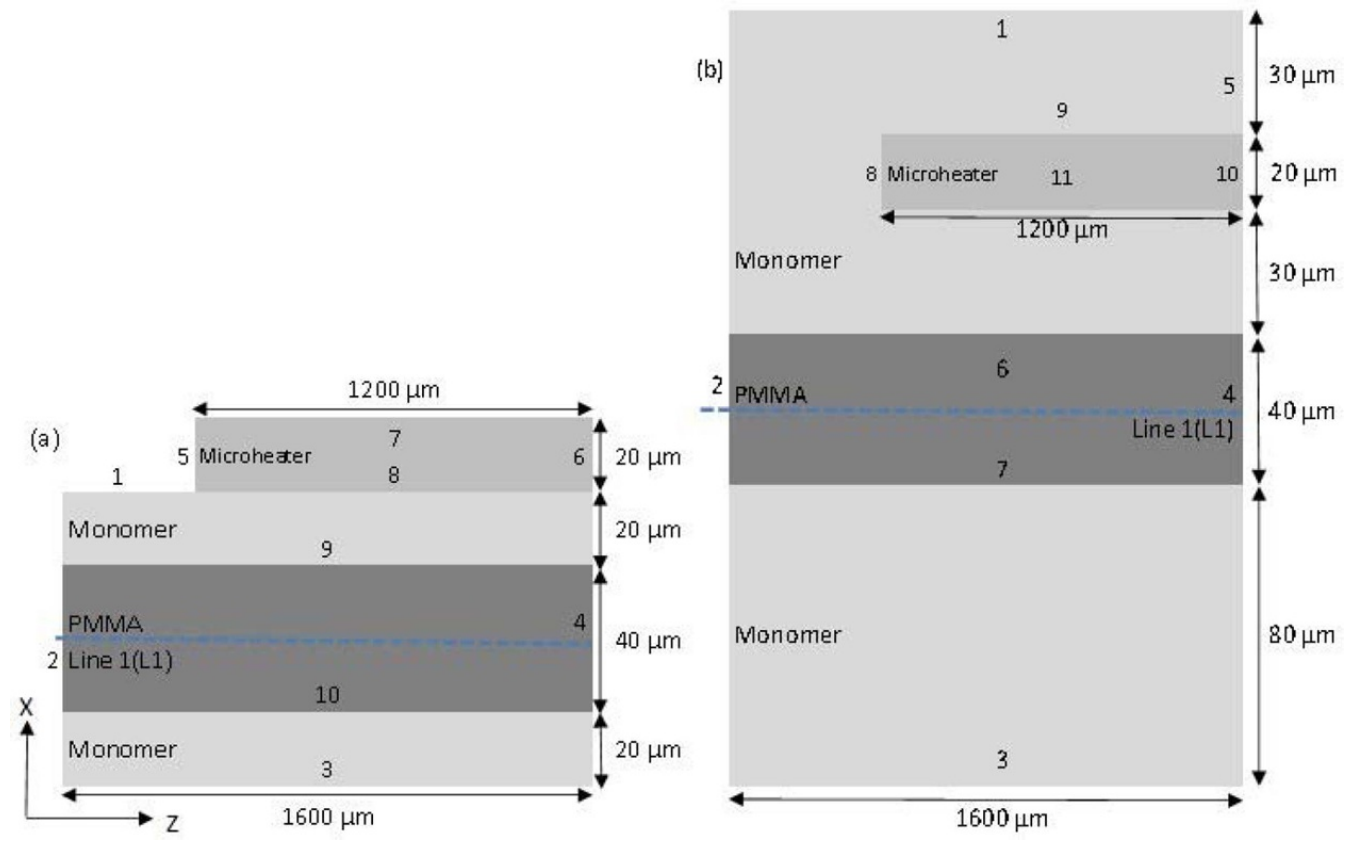

Figure 1. Schematics of the planar PMMA waveguide geometry. (a) Microheater placed at the top of the upper cladding, (b) microheater buried within the upper cladding. The numbers are giving different boundary conditions as explained in the text.

conditions were fixed for thermal analysis. The boundaries denoted as 1,2,3,4 in figure 1 (a) and 1,2,3,4,5 in figure 1(b) were set to a temperature $T_{0}=20^{\circ} \mathrm{C}$. The boundaries denoted as 5,6,7,8 in the first scheme in figure 1 (a) and as 8,9,10,11 in the second scheme in figure $1(\mathrm{~b})$ were set to the temperature of the micro heater $T=T_{\text {microheater. The interface boundaries }}$ between the PMMA core and the monomer cladding medium are fixed as continuity boundary conditions, which establish a link between these media and ensure the smooth flow of heat in-between them. The interior boundaries $(9,10)$ in figure 1 (a) and $(6,7)$ in figure 1 (b) accept this default continuity boundary condition.

The contour maps for the conductive heat transfer distributions within the waveguides when the heater was at the maximum temperature of $200{ }^{\circ} \mathrm{C}$ are shown in figures 2(a) and (b), respectively. The change in temperature in the same layer line L1 along the central propagation $z$-axis for the upper and buried microheater models are shown in figure 3. At this point, the microheater was at the maximum temperature of $200{ }^{\circ} \mathrm{C}$. The temperature along the line $\mathrm{L} 1$ was increased from $20^{\circ} \mathrm{C}$ to $110^{\circ} \mathrm{C}$ for the top microheater on the upper-cladding model. A larger increase in temperature from $20{ }^{\circ} \mathrm{C}$ to $148{ }^{\circ} \mathrm{C}$ was achieved along the line L1 for the buried microheater within the upper cladding model. For both these cases, the microheater was at the temperature of $200^{\circ} \mathrm{C}$. The solver was static for all simulations. However, in one case it was selected time dependent to find the spreading time for the heat throughout the waveguide. In that case, the spreading time was $7 \mathrm{~ms}$. The microheater placed on top of the waveguide cladding provided better and controlled increase in temperature while the embedded microheater could possibly be used to obtain quicker switching times. Also, the temperature distribution along the reference line L1 shows a slightly smoother increase for the top microheater compared to the buried microheater case; see figure 3. It is also more complex to implement a heater within the cladding of a waveguide in real world situations. Also, the modulation in temperature along the line L1 for the top microheater increased gradually when the microheater temperature was increased from $20^{\circ} \mathrm{C}$ to $200{ }^{\circ} \mathrm{C}$; figure 4 . The temperature through the line L1 in the PMMA core part of the waveguide never exceeded the value $110{ }^{\circ} \mathrm{C}$, when the top microheater was at a high temperature of $200{ }^{\circ} \mathrm{C}$. The increase in temperature along the line $\mathrm{L} 1$ when the temperature of the buried microheater was varied between $20^{\circ} \mathrm{C}$ and $200{ }^{\circ} \mathrm{C}$ is provided in figure 5. The increase in temperature along $\mathrm{L} 1$ was proportional to the temperature of the microheater. The buried microheater induced a larger increase in temperature along the line L1 than the heater on the top of the waveguide.

Zhang et al evaluated many polymers including PMMA and demonstrated a linear relationship between the thermo-optic coefficients $(\mathrm{d} n / \mathrm{d} T)$ and the coefficients of thermal expansion $(\alpha)$ [21]. The change in refractive index as a function of temperature was measured for PMMA material, which is also considered in our case [21].

Here, the refractive indices for the core and cladding of the polymer waveguide are calculated by considering the temperature distributions obtained from the thermal simulations. Equation (5) was utilized for this [32].

$$
n_{\text {temp }}=n_{o}+n_{0} * d n / d T *\left(T_{1}-T_{0}\right) .
$$

Here, $\frac{d n}{d T}$ is the thermo-optic coefficient for PMMA and is taken as $\frac{d n}{d T}=-1.3 \times 10^{-4}{ }^{\circ} \mathrm{C}^{-1} . T_{0}=20{ }^{\circ} \mathrm{C}$ is the initial room temperature and $T_{1}$ is the induced increased in temperature due to the heating by the microheater, as obtained 


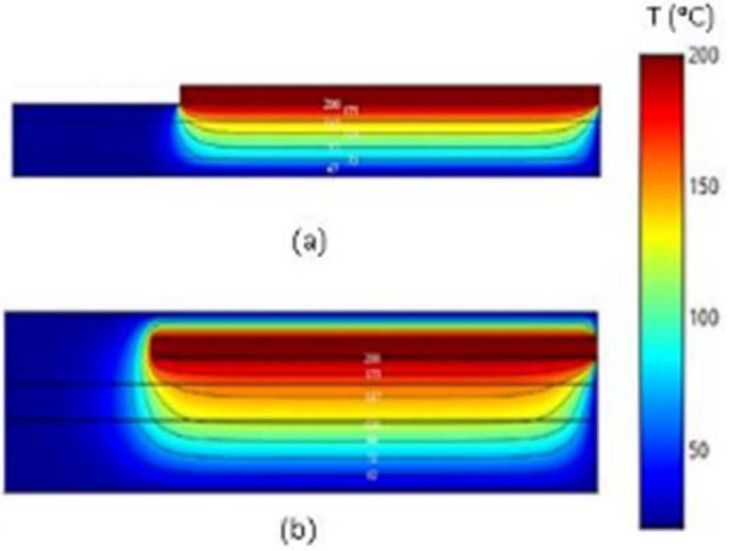

Figure 2. Contour maps for the temperature distribution within the waveguides. (a) Microheater is at the top of the upper cladding, (b) microheater is buried inside the middle region of the upper cladding.

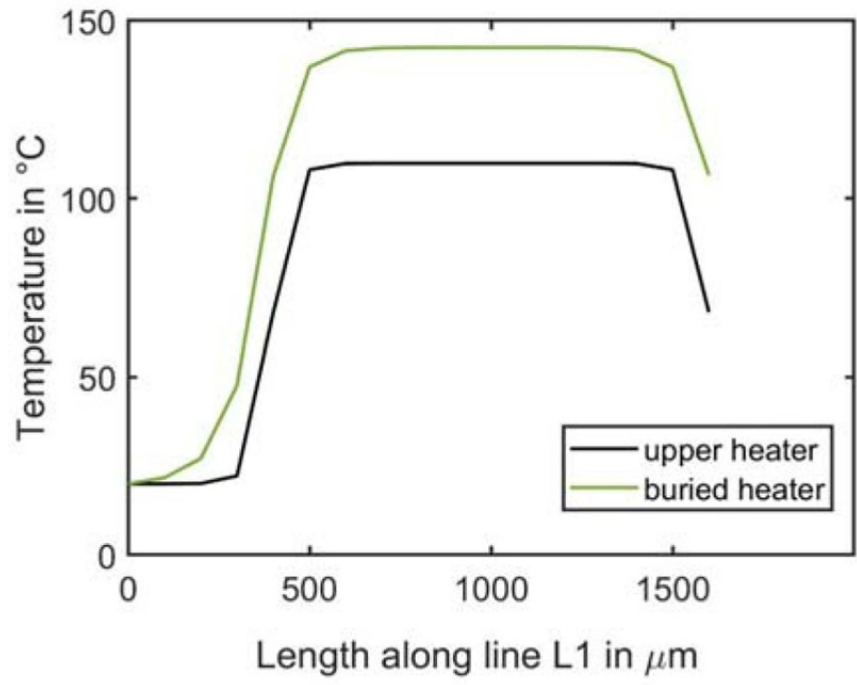

Figure 3. The change in temperature distribution along the line L1 (central core axis along beam propagation direction) for the maximum microheater temperature at $200{ }^{\circ} \mathrm{C}$. (a) Upper heater, (b) buried heater. The heating time was $7 \mathrm{~ms}$.

from the solution of the finite element heat analysis. $n_{0}$ is the initial value of the refractive index at room temperature and $n_{\text {temp }}$ is the new/updated value of refractive index calculated from the change in temperature within the waveguide due to the heating process and the thermo-optical coefficient.

The initial values of refractive indices of the PMMA core and monomer cladding were 1.48 and 1.41 , respectively. The polymer material possesses a negative thermo-optical coefficient, so there is a decrease in the refractive index value with an increase in temperature, as also observed in the simulations. The observed decrease in refractive index of the core of the waveguide was from 1.4885 to 1.4600 , while the temperature of microheater was varied between $20^{\circ} \mathrm{C}$ and $200{ }^{\circ} \mathrm{C}$. The corresponding refractive index of the cladding decreased from 1.412 to 1.392 during the heating process. Also, the associated change in numerical aperture was calculated for

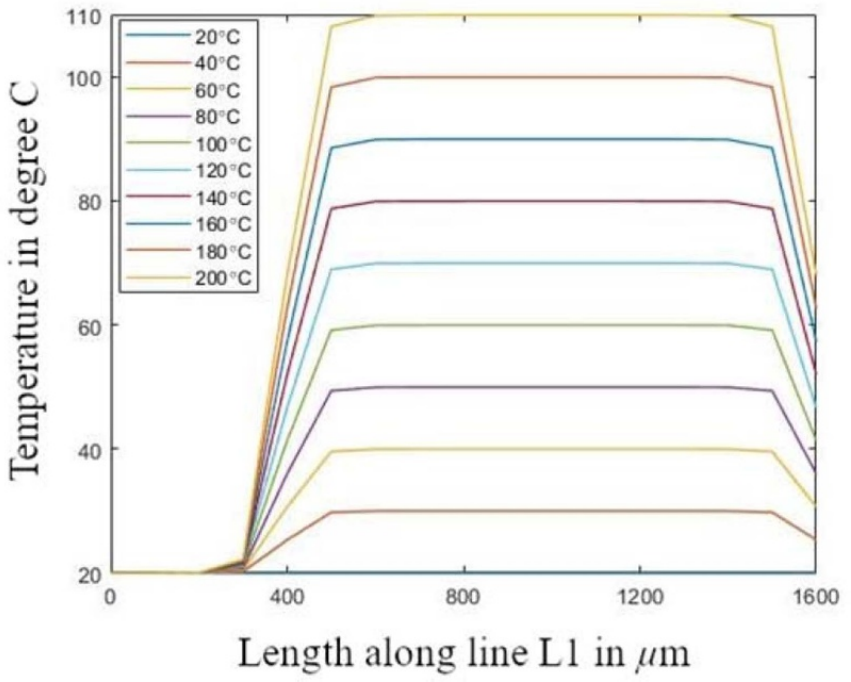

Figure 4. Temperature modulation along the central beam propagation axis $\mathrm{L} 1$ when the temperature of the top microheater was varied between $20^{\circ} \mathrm{C}$ and $200{ }^{\circ} \mathrm{C}$.

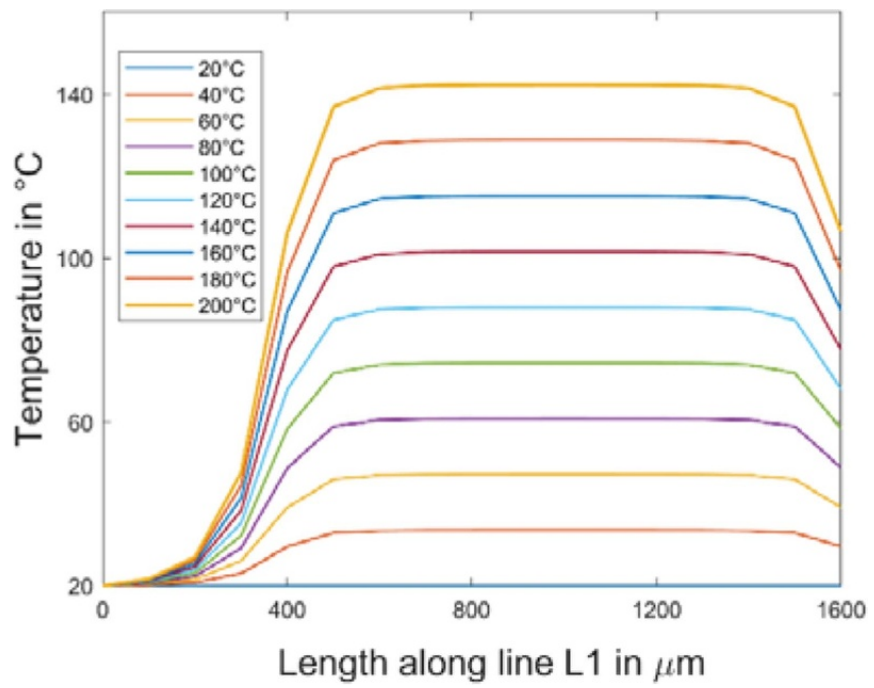

Figure 5. Temperature modulation along the central beam propagation axis $\mathrm{L} 1$ when the temperature of the buried microheater was varied between $20^{\circ} \mathrm{C}$ and $200{ }^{\circ} \mathrm{C}$.

the temperature range considered; see figure 6 . The numerical aperture (NA) of the PMMA waveguide decreased from 0.471 to 0.457 during this process. Here, the NA for the waveguide was calculated by using $\sqrt{n_{\text {core }}^{2}-n_{\text {clad }}^{2}}$. There was a refractive index gradient across the core of the waveguide. However, it was not significant (the size of the gradient across the core was 0.00015 ) due to the smaller core diameter. The refractive index gradient was considered for performing the optical simulations.

Subsequent to the finite element analysis for the thermal distribution, the computational waveguide models were transferred to the CN-BPM simulation (implemented in MATLAB) to carry out the optical simulations. The initial input beam with Gaussian beam profile was coupled into the PMMA 


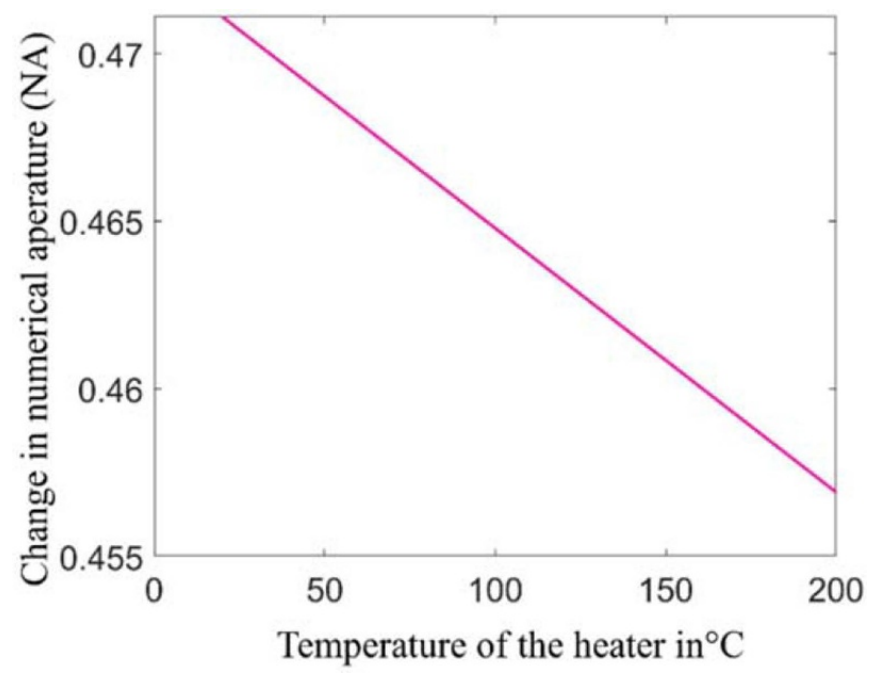

Figure 6. Change in NA of the polymer waveguide as the temperature of the microheater was varied between $20^{\circ} \mathrm{C}$ and $200^{\circ} \mathrm{C}$.

waveguide core. The intensity of the input beam was normalized to the maximum peak value of one. The optical simulation was then carried out at different temperature distribution profiles of the PMMA waveguides imported from the FEATool. The obtained beam intensity distribution profiles are shown in figure 7 , for selected temperatures of the microheater between $60{ }^{\circ} \mathrm{C}$ and $180{ }^{\circ} \mathrm{C}$, respectively. The decrease in the output intensity of waveguide was not significant until the temperature of microheater reached $60{ }^{\circ} \mathrm{C}$. Afterwards, the decrease became more prominent. The primary eyes which are the localized maximum intensity points that indicate the guiding properties of PMMA waveguides tend to move within the waveguide as the light beam propagates within them. Back and forth movements of the primary eyes within the PMMA waveguide cores were observed when the microheater was at selected temperatures of $60^{\circ} \mathrm{C}, 80^{\circ} \mathrm{C}, 100^{\circ} \mathrm{C}$ and $150^{\circ} \mathrm{C}$, as shown in figure 7. This is an indication of changed beam propagation conditions and, in this case, decreased transmitted beam intensity at the output of the waveguide core. The primary eyes almost disappeared when the microheater was at $180{ }^{\circ} \mathrm{C}$. For this temperature, the output beam intensity from the PMMA waveguide dropped to $50 \%$ of the initial beam intensity. The losses in the waveguide are due to the change in NA as well as due to the residual stresses build up during the heating process. However, we assume that the residual stress is less significant due to the small Young's modulus of the polymer material. As the current simulation is a multi-step process, the accumulated error could be larger than the actual physical process. The change in refractive index of the material depends on the thermo-optic coefficient. In addition, the change in refractive index depends on the size of the microheater as well as the size of the wave-guiding element. In the current simulations, the $\frac{d n}{d T}$ value of PMMA was considered for both the core and the cladding part. This could be different for different materials
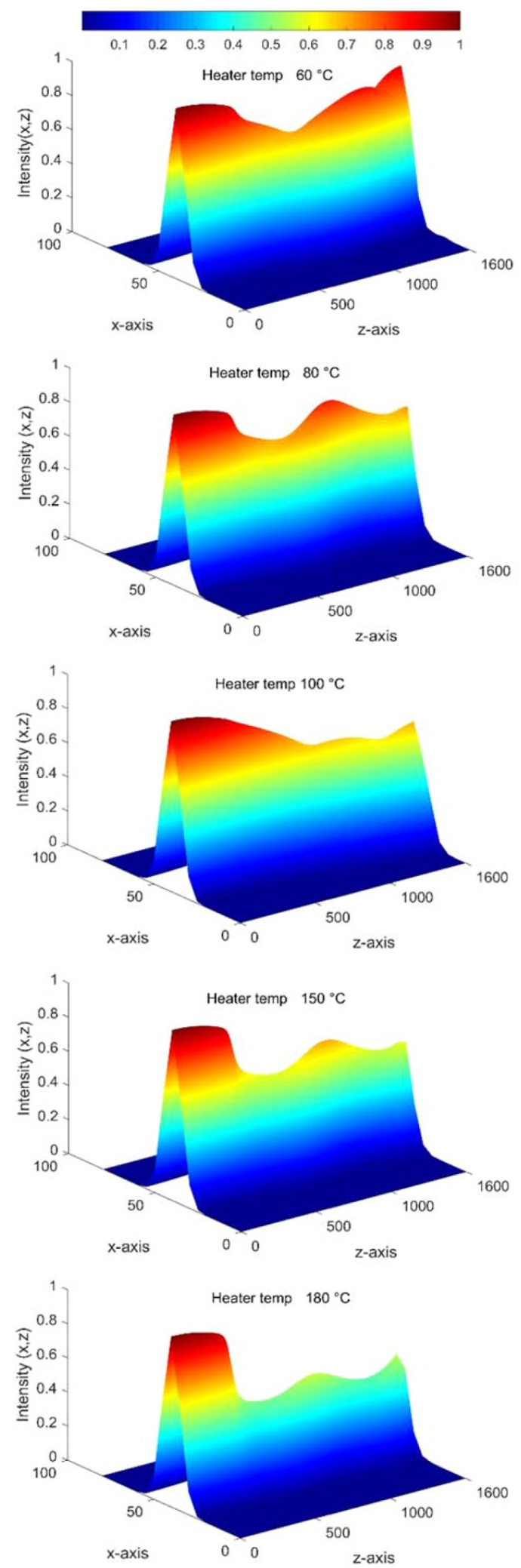

Figure 7. Intensity distribution profiles of the propagating beam within the waveguide at different temperatures for the microheater. Units on the $x$-axis and $z$-axis are given in $\mu \mathrm{m}$. The intensity $I(x, z)$ is normalized to the maximum initial input intensity value 1 . 


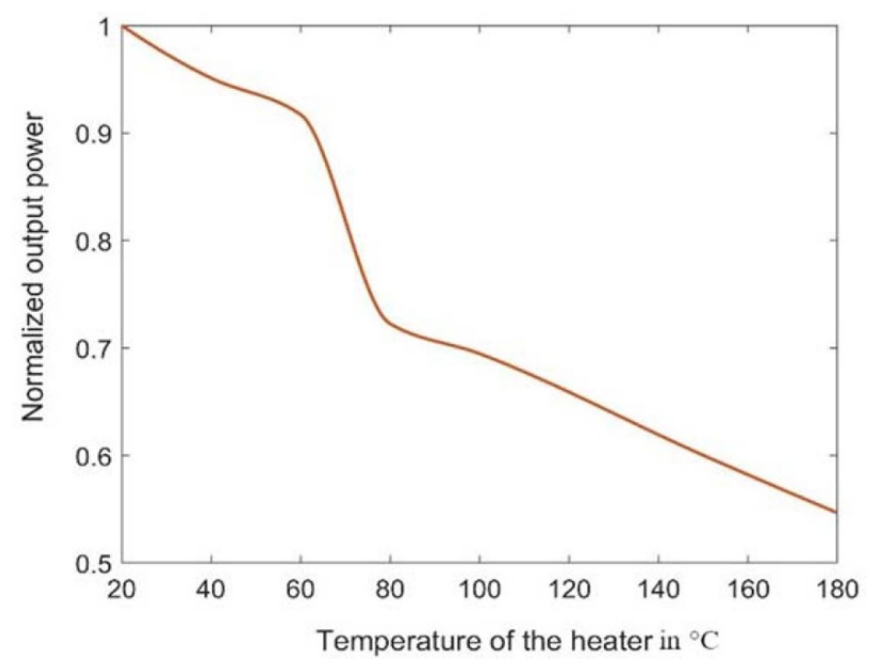

Figure 8. Normalized output power from the exit port of the PMMA waveguide.

and could vary in the actual physical processes. Experimental validation of the simulation is currently being performed to validate the approach. The output power at the exit port of the waveguide was then calculated for each case by considering the last slice of the PMMA waveguide and integrating the intensity values over the area. The obtained output power from the exit port of the waveguide is shown in figure 8 . The normalized output power decreased as the heating by the microheater was increased. A sharper decrease in the output power was observed between $60{ }^{\circ} \mathrm{C}$ and $80^{\circ} \mathrm{C}$. At this point, the PMMA material approaches the glass transition temperature [33]. The output power continued to decrease further and at $180{ }^{\circ} \mathrm{C}$ the $P_{\text {output }}=0.54 * P_{\text {input }}$. When the temperature of the heater is increased to even higher temperatures, the decrease in refractive indices of core and cladding will be larger and finally the beam would not be confined to the waveguide core anymore, but diffuse to the surrounding media.

The deformation or elongation produced in polymer based integrated photonic device is an important consideration to access their performance in varying environmental conditions. Thus, for the integrity of the performance, a link was established between the thermal distribution and stress analysis module in the FEATool. The corresponding elongations/deformations generated in the waveguide are quantified. The finite analysis solver of the Multiphysics FEATool was unitized for the stress and deformation analysis. In particular, the plane stress module with proper boundary conditions was employed for the deformation analysis of PMMA waveguides. The left and right extremities of the waveguide were fixed for the model in figure 1(a). The boundary conditions such as fixing one end or both ends in deformation analysis are crucial. Fixing both ends of the waveguide is a better practical approach as optical waveguides and interconnects are usually placed between fixed active components (LDs or waveguides) in integrated photonic circuits. The heat transfer module was coupled to the plane stress module of the FEATool to perform the thermo-mechanical multiphysics simulations.
Table 2. Model parameter values for stress analysis.

\begin{tabular}{lccr}
\hline & $E(\mathrm{GPa})$ & $\nu$ & $\alpha\left(\mathrm{K}^{-1}\right)$ \\
\hline Monomer & 3.3 & 0.40 & $72.22^{*} \mathrm{e}-6$ \\
PMMA & 2.76 & 0.37 & $22.25^{*} \mathrm{e}-6$ \\
Microheater (polysilicon) & 160 & 0.22 & $2.6 \mathrm{e}^{*} \mathrm{e}-6$ \\
\hline
\end{tabular}

(a)

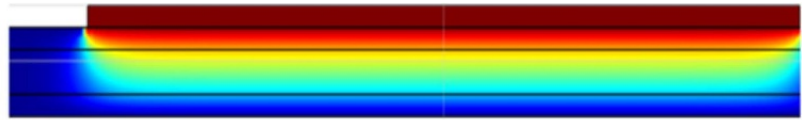

(b)

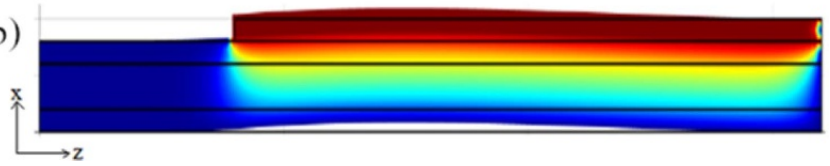

Figure 9. (a) Initial PMMA waveguide at $40{ }^{\circ} \mathrm{C}$, (b) deformed PMMA waveguide at $200{ }^{\circ} \mathrm{C}$.

Here, the initial temperature of the waveguide was again considered as $20^{\circ} \mathrm{C}$. The parameters used for the stress analysis are provided in the table 2 .

In table 2, $\rho$ is the material density, $E$ is its Young's modulus and $\nu$ is the Poisson's ratio signifying the ratio between the transverse contraction strain to the longitudinal extension strain in the direction of the stretching force. The thermal expansion coefficient is denoted by $\alpha$. As can be expected, the maximum deformation was obtained when the microheater was at the temperature of $200{ }^{\circ} \mathrm{C}$. The initial waveguide without deformation (when the microheater was at $40{ }^{\circ} \mathrm{C}$ ) is shown in figure 9(a), whereas the deformed waveguide (when the microheater was at $200{ }^{\circ} \mathrm{C}$ ) is given in figure 9(b). The deformation analysis for the microheater at $200{ }^{\circ} \mathrm{C}$ is presented in figure 10. The elongations along the transverse $x$-axis and the beam propagation $z$-axis are shown in figures 10 (a) and (b), respectively. We obtained a maximum elongation $\left(\Delta L_{x}\right)$ of $831 \mathrm{~nm}$ along $x$-axis and a maximum elongation along $z$-axis $\Delta L_{z}=1397 \mathrm{~nm}$ when the microheater was at $200{ }^{\circ} \mathrm{C}$. The elongations produced along the $x$ - and $z$-axis are small compared to the original waveguide dimensions. The residual stress build-up in the waveguide is assumed to be less severe because of the small Young's modulus of the polymer material. The effect of residual stress is thus not included in the current BPM simulation.

\section{Conclusion}

A combined simulation approach for the thermo-mechanical and optical study of polymer waveguide structures was carried out by incorporating a polysilicon microheater into a waveguide model. The microheater was located on top of or inside the upper cladding of the polymer PMMA waveguide. A finite element analysis was performed by using the FEATool box to obtain the heat distribution and mechanical deformation within the waveguides. The finite difference approach was adapted and implemented into a CN-BPM model in MATLAB. The simulation results obtained from the FEATool box 


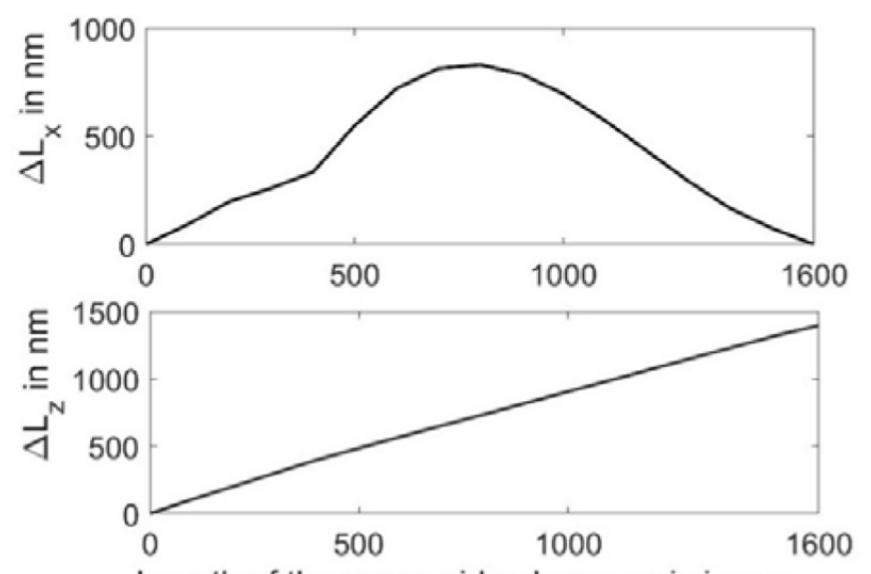

Length of the waveguide along $\mathrm{z}$-axis in $\mu \mathrm{m}$

Figure 10. Deformation in the PMMA waveguide when the heater was at $200{ }^{\circ} \mathrm{C}$. (a) Displacement of the line L1 along the transverse $x$-axis: $\Delta L_{x}=831 \mathrm{~nm}$, (b) displacement of the line L1 along the $z$-axis (propagation direction of the beam): $\Delta L_{z}=1397 \mathrm{~nm}$.

were coupled back to the MATLAB module. The obtained modulation in temperature distribution was found to affect the light beam intensity distribution within the PMMA waveguide significantly, as the polymer material is very sensitive to environmental changes. The refractive indices of core and cladding were computed from the thermal distributions by considering their influence on the thermo-optic coefficients. Due to the heating, the NA of the polymer PMMA waveguide was observed to decrease from 0.471 to 0.457 . The obtained beam intensity profiles within the PMMA waveguide at various microheater temperatures were determined. The movement of maximum intensity primary eyes signifying the beam guiding properties of the waveguide was no longer visible when the temperature of the microheater was increased beyond $180{ }^{\circ} \mathrm{C}$. The normalized output power from the exit port of the waveguide decreased to $0.54 * P_{\text {input }}$ when the microheater reached $180{ }^{\circ} \mathrm{C}$. Consequently, the normalized output power from the exit port of the waveguide is also varied during the heating. No significant change in the output power occurred until $60{ }^{\circ} \mathrm{C}$. Furthermore, for the model dimensions considered here, the deformation of the PMMA waveguide was found to be $831 \mathrm{~nm}$ along the transverse $x$-direction $\left(\Delta L_{x}\right)$ and $1397 \mathrm{~nm}$ along the beam propagation $z$-direction $\left(\Delta L_{z}\right)$ when keeping the waveguide end facets at fixed locations. The study performed helps us to understand the relevant effects and dynamics of PMMA waveguides as a function of relevant parameters and conditions. The modulation of the refractive index with respect to the varied temperature load is a critical aspect for thermo-optical switches or future polymer-based planar sensor networks. More complex PMMA thermo-optic switches and PMMA photonic components will be designed and simulated for thermomechanical analysis and optical simulations in future. Also, systematic experimental studies will be initiated to verify the theoretical findings and eventually extend and optimize the simulations.

\section{Acknowledgments}

We acknowledge support from the Deutsche Forschungsgemeinschaft (DFG) under Germany's Excellence Strategy within the Cluster of Excellence PhoenixD (Photonics, Optics, and Engineering Innovation Across Disciplines), (EXC 2122, projectID 390833453).

\section{ORCID iD}

Monali Suar (D) https://orcid.org/0000-0001-5409-4549

\section{References}

[1] Jang C and Chen R 2003 Polymer-based $1 \times 6$ thermooptic switch incorporating an elliptic TIR waveguide mirror $J$. Lightwave Technol. 211053

[2] Hauffe R, Siebel U, Bruns J and Petemann K 2001 Digital optical circuit switches and switching matrices in polymers Photon. Technol. Lett. 55 305-12

[3] Noh Y, Lee C, Kim J, Hwang W, Won Y, Lee H, Han S and Oh M 2004 Polymer waveguide variable optical attenuator and its reliability Opt. Commun. $242533-40$

[4] Lu Y, Guo H, Wang H and Chi S 2003 Polymer-based $\mathrm{S}$-shaped waveguide VOA for applications in the broadband DWDM network Microw. Opt. Technol. Lett. $391-4$

[5] Ahn S, Steier W, Kuo Y, Oh M, Lee H, Zhang C and Fetterman H 2002 Integration of electro-optic polymer modulators with low-loss fluorinated polymer waveguides Opt. Lett. 27 2109-11

[6] Suar M, Melchert O, Rahlves M and Roth B 2019 Experimental and theoretical study of the formation process of photopolymer based self-written waveguides $O p t$. Express 27 38326-36

[7] Suar M, Rahlves M, Reithmeier E and Roth B 2018 Numerical investigations on polymer-based bent couplers J. Opt. Soc. Am. B 35 1896-904

[8] Ma C, Zhang H, Zhang D, Cui Z and Liu S 2004 Effects of trapezoid core cross-sections on transmission characteristics of polymer arrayed waveguide grating multiplexers $O p t$. Commun. 241 321-31

[9] Keil N, Yao H and Zawadzki C 2001 A thermal polarisation-independent arrayed-waveguide grating (AWG) multiplexer using an all-polymer approach Appl. Phys. B 73 619-22

[10] Rahlves M, Günther A, Rezem M and Roth B 2018 Polymer-based transmission path for communication and sensing applications J. Lightwave Technol. 37 729-35

[11] de Felipe D, Kleinert M, Zawadzki C, Polatynski A, Irmscher G, Brinker W, Moehrle M, Bach H-G, Keil N and Schell M 2017 Recent developments in polymer-based photonic components for disruptive capacity upgrade in data centers J. Lightwave Technol. 35 683-9

[12] Malallah R, Cassidy D, Muniraj I, Ryle J P, Healy J J and Sheridan J T 2018 Self-written waveguides in photopolymer Appl. Opt. 57 E80-8

[13] Malallah R, Li H, Muniraj I, Cassidy D, Al-attar N, Healy J J and Sheridan J T 2018 Controlling the trajectories of self-written waveguides in photopolymer J. Opt. Soc. Am. B 35 2046-56

[14] Günther A, Schneider S, Rezem M, Wang Y, Gleissner U, Hanemann T, Overmeyer L, Reithmeier E, Rahlves M and Roth B 2017 Automated misalignment compensating 
interconnects based on self-written waveguides $J$.

Lightwave Technol. 35 2678-84

[15] Rezem M, Günther A, Roth B, Reithmeier E and Rahlves M 2017 Low-cost fabrication of all-polymer components for integrated photonics J. Lightwave Technol. 35 299-308

[16] Rezem M, Günther A, Rahlves M, Roth B and Reithmeier E 2014 Hot embossing of polymer optical waveguides for sensing applications Procedia Technol. 15 514-20

[17] Horvath R, Pedersen H C, Skivesen N, Svanberg C and Larsen N B 2005 Fabrication of reverse symmetry polymer waveguide sensor chips on nanoporous substrates using dip-floating J. Micromech. Microeng. 15 1260-4

[18] Kelb C, Pätzold W, Morgner U, Rahlves M, Reithmeier E and Roth B 2016 Characterization of femtosecond laser written gratings in PMMA using a phase-retrieval approach $O p t$. Mater. Express 6 3202-9

[19] Li H, Qi Y, Malallah R and Sheridan J T 2015 Modeling the nonlinear photoabsorptive behavior during self-written waveguide formation in a photopolymer J. Opt. Soc. Am. B 32 912-22

[20] Tong X C 2013 Advanced Materials for Integrated Optical Waveguides (Bern: Springer)

[21] Zhang Z, Zhao P, Lin P and Sun F 2006 Thermo-optic coefficients of polymers for optical waveguide applications Polymer 47 4893-6

[22] Hauffe R and Petermann K 2006 Thermo-optic switching Optical Switching ed T S El-Bawab (Boston, MA: Springer)

[23] Kasarova S, Sultanova N and Nikolov I D 2010 Temperature dependence of refractive characteristics of optical plastics J. Phys.: Conf. Ser. 253012028

[24] Rahman M and Hossain M 2014 Thermal performance analysis of polymer optical waveguides for thermo-optic applications Int. Forum on Strategic Technology (IFOST) pp 65-8

[25] Liu A, Zhang Z, Liu D, Keil N and Grote N 2013 Thermo-optic simulations of silicon nitride/polymer hybrid waveguides Proc. SPIE 8781878105

[26] Aronow R et al 2017-2018 Multiple scale modeling for predictive material deformation analysis Math. Stat. Newsl. 33 4-6

[27] Shewchuk J 1970 Triangle: engineering a 2D quality mesh generator and delaunay triangulator Applied Computational Geometry: Towards Geometric Engineering pp 203-22 (https://doi.org/10.1007/BFb0014497)

[28] Samah A 2004 Design and fabrication of a polymer based directional coupler thermooptic switch PhD Thesis Universiti Teknologi Malaysia, Faculty of Electrical Engineering

[29] Ibrahim M, Kassim N, Mohamad A B and Kamsah N 2007 Thermal analysis in optical waveguides J. Teknologi 46 93-102

[30] Pedrola G L 2015 Beam Propagation Method for Design of Optical Waveguide Devices (New York: Wiley)

[31] Hadley G R 1992 Transparent boundary condition for the beam propagation method IEEE J. Quantum Electron. $28363-70$

[32] Diemeer M, Brons J and Trommel E 1989 Polymeric optical waveguide switch using the thermooptic effect $J$. Lightwave Technol. 7 449-53

[33] Dixit M, Mathur V, Gupta S, Baboo M, Sharma K and Saxena N 2009 Morphology, miscibility and mechanical properties of PMMA/PC blends Phase Transit. Multinational J. 82 866-78 